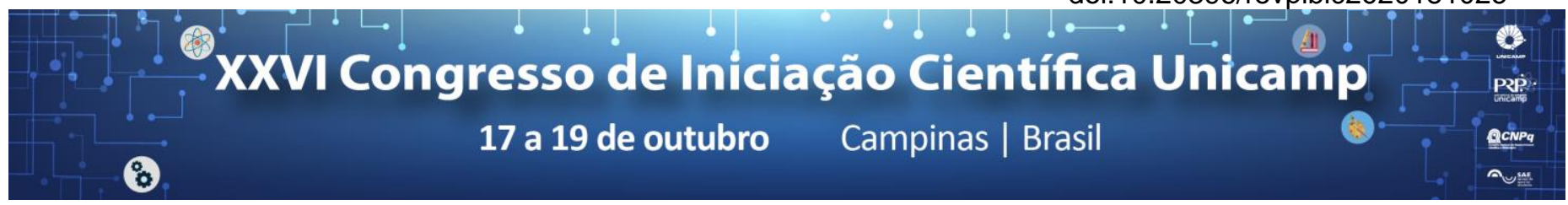

\title{
Application of enantioselective Heck-Matsuda reactions in the synthesis of densely functionalized cyclopentenols.
}

\section{Matheus Aquilini, Ricardo A. Angnes, Carlos R. D. Correia.}

\begin{abstract}
With the progress on the enantioselective arylation of 3-cyclopentenol obtained by our group, the present project was motivated to explore new possible substrates for the enantioselective Heck-Matsuda reactions (EHM) and try to propose new synthetic routes for tricyclic skeletons, found in tromboxan inhibitors and other related pharmaceuticals. In this project, 2-substituted derivatives of 3-cyclopentenol were prepared from cyclopentadiene epoxide using substituents like ethyl, cyanomethyl and esthers, and evaluated as substrates for EHM. After evaluation and optimazation of reaction conditions, it was possible to obtain satisfactory yields, isolate and characterize different arylation products using techniques as HPLC, GCMS and NMR, providing a better understanding of the reaction scope and EHM applications.
\end{abstract}

Key words: Palladium, catalysis, enantioselective.

\section{Introduction}

Heck reactions promote the formation of $\mathrm{C}-\mathrm{C}$ bonds using transition metal complexes and were first reported in the 60 s by Richard F. Heck ${ }^{1}$. Thereafter, variations were developed and allowed for the use of more stable and less toxic reagents under less demanding conditions $^{5}$. Heck-Matsuda reactions promote the coupling of aryl groups to olefins using aryldiazonium salts and palladium complexes in presence of base ${ }^{2}$.

Through the use of chiral ligands in the complex and benefiting from non-covalent interactions between the olefin and the palladium center, our group managed to rationalize the interactions that rule the catalytic cycle and to promote the enantioselective arylation of many olefins, amongst them 3-cyclopentenol ${ }^{3}$.

Cyclopenta[b]benzofuran skeletons, obtainable from related phenolic arylcyclopentenols 6,7 , are found in a variety of bioactive compounds and pharmaceuticals ${ }^{4}$. In biological systems, the identification and functionality of molecules are greatly dependent on their stereochemistry, thus, the enantioselective catalysis explored in this project may have applications on the design and discovery of new pharmaceuticals and synthetic routes.

The structures of the olefins used as EHM substrates were chosen in order to obtain useful information about the behavior of different 3-cyclopentenol derivatives under EHM as well as arylation products that could be further derived into interesting structures.

\section{Results and Discussion}

The olefins studied in this project were synthesized from cyclopentadiene epoxide, which was prepared from commercial dicyclopentadiene. The initial EHM test were carried at $0,1 \mathrm{mmol}$ scale, varying parameters like the charge of palladium catalyst, different ligands, aryldiazonium salts, mixtures of solvents, bases and stoichiometry. The arylation products were then isolated by chromatography and characterized using NMR and GCMS, while chiral HPLC analysis provided the enantiomeric excess (ee) in which the products were obtained.

Varying the cited parameters and analyzing the yield and ee obtained with each combination, it was possible to optimize reaction conditions and obtain arylation yields in the $70-80 \%$ range as well as ee's of $90 \%$ in some cases.

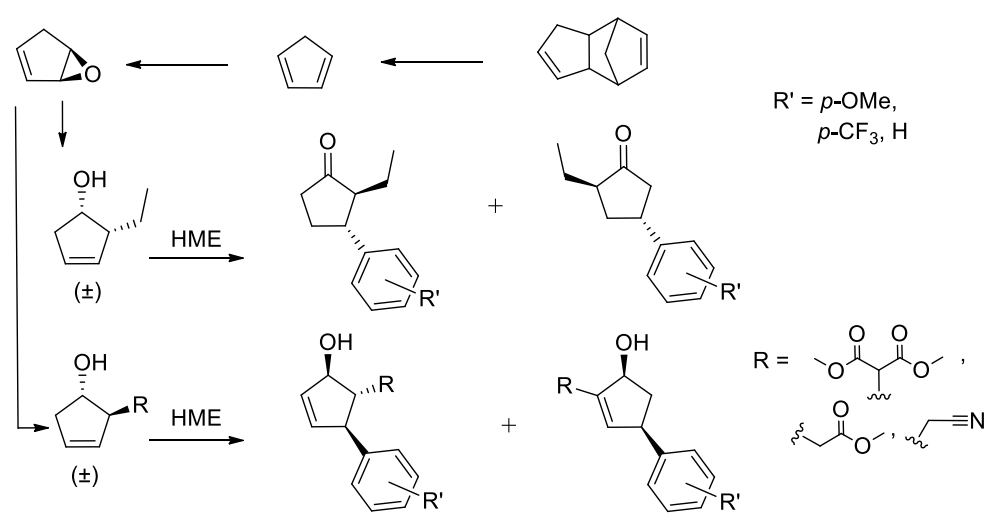

Image 1. General scheme of the synthetic steps performed.

Electronic properties of the substituent at position 2 may alter the arylation site and obtained products ${ }^{8}$. It was observed by NMR that its geometry may also affect that of the arylated carbon, possibly due to steric hindrance between the substituent and the ligand in the complex.

Some of the products obtained as mixture of diastereoisomers could not be isolated in regular or AgNO3-doped silica, only under chiral HPLC, and did not have their structure fully elucidated. Analogues of these products were synthesized by altering the $R$ and $R^{\prime}$ chains, leading to the formation of products that could be isolated using chromatography and then characterized.

\section{Conclusions}

During project development, it was possible to start from dicyclopentadiene, an abundant by-product of oil cracking, and synthesize substrates to study Heck-Matsuda reactions, yielding valuable structures, methodologies and a better understanding of the behavior 3-cyclopentenol derivatives under EHM conditions.

\section{Acknowledgement}

To CNPq for the given support during development.

${ }^{1}$ Heck, R. F. J. Am. Chem. Soc. 1968, 90 (20), 5526.

${ }^{2}$ Kikukawa, K.; Matsuda, T. Chem. Lett. 1977, 6 (2), 159.

${ }^{3}$ Angnes, R. A.; Oliveira, J. M.; Oliveira, C. C.; Martins, N. C.; Correia, C. R.

D. Chem. Eur. J. 2014, 20 (41), 13117.

${ }^{4}$ Melian, E. B.; Goa, K. L. Drugs 2012, 62 (1), 107.

${ }^{5}$ Beletskaya, I. P.; Cheprakov, A. V. Chem. Rev. 2000, 100 (8), 3009.

${ }^{6}$ Aponick, A.; Li, C.-Y.; Biannic, B. Org. Lett. 2008, 10, 669

${ }^{7}$ Correia, C. R. D. et al. J. Org. Chem. 2016, 81 (5), 2010.

${ }^{8}$ Sigman, M. S. et al. J. Am. Chem. Soc. 2015, 137 (23), 7290. 